\title{
NUMERICAL METHODS OF SOLVING ONE-DIMENSIONAL NON-STATIONARY GAS-DYNAMIC PROBLEMS*
}

\author{
Yu. P. POPOV and A. A. SAMARSKII
}

Moscow

(Received 24 March 1976)

\begin{abstract}
A DESCRIPTION is given of difference schemes for systems of one-dimensional non-stationary gas dynamic equations, constructed on the basis of the principles of conservativeness, complete conservativeness, and homogeneity, and permitting coarse meshes to be used. A comparative analysis of various methods of solving difference schemes representing systems of non-linear algebraic equations is presented. It is shown that Newton's iterative method permits the use of meshes with the greatest time steps.
\end{abstract}

\section{Introduction}

Many problems of modern science and technology include as a basic element the equations of gas dynamics. These equations are non-linear, and at the present time the only efficient and universal methods of solving them are numerical methods based on the use of high-speed computers.

In actual problems of practical interest the equations of gas dynamics are as a rule complicated by the introduction of additional factors, such as, for example, electromagnetic fields, thermal and electrical conduction processes, chemical reactions, radiation etc. The development of algorithms for the solution of such complicated systems of equations presents considerable difficulties, and their realization requires a large amount of computer time. Therefore the development of efficient methods of solving the gas dynamic equations numerically, which would permit the use of "coarse" difference meshes, is of great importance.

We explain the problems existing here and possible methods of solving them by the example of a system of homogeneous non-stationary equations of gas dynamics in Lagrangian mass coordinates. For the case of plane symmetry this system of equations has the form $[1,2]$

$$
\begin{gathered}
\frac{\partial v}{\partial t}=-\frac{\partial p}{\partial s}, \\
\frac{\partial x}{\partial t}=v, \\
\frac{\partial}{\partial t}\left(\frac{1}{\rho}\right)=\frac{\partial v}{\partial s}, \\
\frac{\partial}{\partial t}\left(\varepsilon+\frac{v^{2}}{2}\right)=-\frac{\partial}{\partial s}(p v),
\end{gathered}
$$

"Zh. vychisl, Mat. mat. Fiz., 16, 6, 1503-1518, 1976. 


$$
p=\mathscr{P}(\rho, T), \quad \varepsilon=\mathscr{E}(\rho, T)
$$

Here $t$ is the time, $x$ is the Eulerian variable, $\rho$ is the density of the medium, $s(d s=\rho d x)$ is the Lagrangian mass coordinate, $v$ is the velocity, $p$ is the pressure, $\epsilon$ is the internal energy, and $T$ is the temperature of the gas; the derivatives with respect to time are Lagrangian.

Relations (1.5), closing the system of equations, are the equations of state; their form is determined by the properties of the medium considered in a specific problem.

\section{Homogeneous completely conservative difference schemes}

In the solution of problems of gas dynamics by the method of finite differences the continuous medium is replaced by some discrete model, and accordingly, the system of differential equations by some difference scheme. The difference scheme approximating the original system of differential equations may be constructed non-uniquely. Therefore some preference criteria are necessary which will enable us to choose from the family of permissible schemes the schemes possessing the best characteristics.

At the present time a number of general characteristics exist (conservativeness, complete conservativeness, homogeneity etc. [2]), which make it possible to construct for Eqs. (1.1)-(1.5) schemes correctly yielding solutions even on coarse meshes, when the approximation is practically lost. These principles are (mainly) of a qualitative nature, they have a theoretical justification for the linear case and are confirmed by practical calculations for non-linear problems.

The application of these principles enables us to construct for the system of equations (1.1) - (1.5) the following one-parameter family of difference schemes:

$$
\begin{gathered}
v_{t}=-\bar{p}_{\bar{s}}^{(\sigma)}, \\
x_{t}=v^{(0.5)}, \\
(1 / \bar{\rho})_{t}=v_{s}^{(0.5)}, \\
\left(\bar{\varepsilon}+\frac{v^{2}+v^{2}(+1)}{4}\right)_{t}=-\left(\bar{p}^{(\sigma)} v^{(0.5)}\right)_{s}, \\
\bar{p}=\mathscr{P}(\bar{\rho}, \bar{T}), \quad \bar{\varepsilon}=\mathscr{E}(\bar{p}, \bar{T}) .
\end{gathered}
$$

For simplicity the scheme is written on the uniform mesh $\Omega=\left\{\left(s_{i}, t_{j}\right), i=0,1, \ldots, N ; j=0,1, \ldots\right.$, $\left.s_{i+1}=s_{i}+h, t_{j+1}=t_{j}+\tau\right\}, h, \tau=$ const are the mesh steps. The mesh functions $x_{j}=x_{i}{ }^{j}, v=v_{i}{ }^{j}$ are referred to the nodes of the mesh $\left(s_{i}, t_{j}\right)$; the functions $\bar{p}=p_{i+1 .}^{j}, \bar{\rho}=\rho_{i+1 / 2}^{j}, \bar{\varepsilon}=\varepsilon_{i+1 / 2}, \bar{T}=T_{i+1 / 2}^{j}$ to the "half-integral" points $\left(s_{i+1 / 2}, t_{j}\right)$, where $s_{i+1 / 2}=s_{i}+h / 2$. The parameter $0 \leqslant \sigma \leqslant 1$ is arbitrary. In the formulas of the scheme (2.1)-(2.5) the following subscriptless notation is used $[2,3]$ : 


$$
\begin{aligned}
& y=y_{i}^{i}, \quad \vec{y}=y_{i+1 / 2}^{j}, \quad \hat{y}=y_{i}^{j+1}, \quad y( \pm 1)=y_{i \pm 1}^{j}, \\
& y_{*}=\frac{y(+1)-y}{h}, \quad y_{\bar{s}}=\frac{y-y(-1)}{h}, \quad y_{t}=\frac{\hat{y}-y}{\tau}, \\
& y_{*}=0.5(y+y(-1)), \quad y^{(\sigma)}=\sigma \hat{y}+(1-\sigma) y .
\end{aligned}
$$

In the scheme (2.1)-(2.5) the laws of conservation of momentum and energy are satisfied. This follows from the divergent form of the difference equations (2.1) and (2.4), which in fact express the laws indicated for one mass interval of the mesh $h$ after one time step $\tau$. The corresponding integral conservation laws are obtained by summation over the mesh of Eqs. (2.1) and (2.4) for $0 \leqslant i \leqslant N, j_{1} \leqslant j \leqslant j_{2}$.

The law of conservation of mass is automatically satisfied in the scheme because of the use of Lagrangian mass variables.

The internal energy balance is also satisfied in the scheme (2.1)-(2.5). Indeed, from the energy equation (2.4) taking into account (2.1)-(2.3) we can obtain the relations

$$
\bar{\varepsilon}_{t}=-\bar{p}^{(\sigma)} v_{\bar{s}}^{(0.5)}, \quad \bar{\varepsilon}_{t}=-\bar{p}^{(\sigma)}(1 / \bar{\rho})_{t},
$$

which express this balance for one cell of the mesh. The difference equations (2.7) approximate the differential equations

$$
\frac{\partial \varepsilon}{\partial t}=-p \frac{\partial v}{\partial s}, \quad \frac{\partial \mathfrak{e}}{\partial t}=-p \frac{\partial}{\partial t}\left(\frac{1}{\rho}\right)
$$

which are consequences of Eqs. (1.1)-(1.4) and reflect the fact that the internal energy of the gas is changed because of the work of the pressure forces.

The equations of the scheme (2.2), (2.3) imply the natural equation

$$
1 / \bar{p}=x_{a},
$$

or, after summation over the mesh,

$$
\sum_{i=0}^{N-1} \frac{h}{\rho_{i+1 / 2}^{j}}=x_{N}^{j}-x_{0}{ }^{j}
$$

The relations (2.8), (2.9) approximate the equations

$$
\frac{1}{\rho}=\frac{\partial x}{\partial s}, \quad \int_{0}^{M} \frac{d s}{\rho}=x(M, t)-x(0, t),
$$

which follow from (1.2), (1.3) and express the so-called law of conservation of volume ( $M$ is the mass of the gas considered in the problem, arriving on unit area of the transverse section) $[1,2]$. 
Therefore, the scheme (2.1)-(2.5) possesses the property of complete conservativeness $[2,4]$. In it are satisfied not only the difference analogs of the fundamental laws of conservation (of mass, momentum, and energy), but also the additional relations (2.7), (2.8), the need for whose observance is dictated by physical considerations.

We note that scheme $(2.1)-(2.5)$ simulates relations $\left(2.7^{\prime}\right),(2.10)$ for any values of the mesh steps $h$ and $\tau$. Schemes which are not completely conservative possess such a property only in the asymptotic sense as $\tau \rightarrow 0$. The violation of the property of complete conservativeness leads to the occurrence in the scheme of various sources of energy of difference origin, whose power is proportional to the mesh step $\tau$. On coarse meshes for large values of $\tau$ these sources are significant and may considerably distort the solution [2].

In practice in the scheme (2.1)-(2.5) instead of the divergent difference equation of energy (2.4) one of the equations (2.7) equivalent to it is often used, and instead of the continuity equation (2.3), the more compact form (2.8).

The approximation error of the scheme (2.1)-(2.5) is $O\left(\tau+h^{2}\right)$ and $O\left(\tau^{2}+h^{2}\right)$ for the particular case of the parameter $\sigma=0.5$.

To ensure homogeneity of the scheme, that is, the possibility of straight through calculation of shock waves without the explicit isolation of the wave front in the mesh, the pseudoviscosity $\bar{\omega}[2,5]$ is introduced into the scheme in the form of an addition to the pressure $\bar{p}$. The presence of pseudoviscosity in the scheme does not violate its complete conservativeness. The corresponding difference scheme is identical with (2.1)-(2.5) if the pressure $\bar{p}$ in Eqs. (2.1) and (2.4) is replaced by the complete pressure $\bar{g}=\bar{p}+\bar{\sigma}$.

In the solution of actual problems the difference equations (2.1)-(2.5) are supplemented by a difference approximation of the boundary and initial conditions.

\section{An explicit completely conservative scheme}

The difference scheme (2.1)-(2.5) is a system of non-linear algebraic equations in the values of the mesh functions. The number of equations in the system is determined by the number of mesh nodes in the spatial variable and may be considerable (30 to 200). Therefore in the general case the solution of such a system represents an independent problem.

In order to analyze the theoretical aspect of the question we confine ourselves to a consideration of only the isothermal approximation. In this case the energy equation is replaced by the relation

$$
T=T_{0}=\text { const, }
$$

which noticeably simplifies the problem. We also note that the use of the method of successive pivotal condensations $[2,8]$ splits the complete system of equations $(2.1)-(2.5)$ into parts, solved separately; then in the dynamic part the equations for the isothermal case in fact occur.

The difference scheme (2.1)-(2.5) in the isothermal approximation, taking into account (2.8), reduces to the form

$$
v_{i}=-p_{\bar{s}}^{(0)}
$$




$$
\begin{gathered}
x_{l}=v^{(0.5)}, \\
(1 / \rho)_{t}=v_{s}^{(0.5)}, \\
p=\mathscr{P}(\rho) .
\end{gathered}
$$

Here and below the bar above the functions $p, \rho$, indicating that they refer to the half-integral points of the mesh, are omitted.

As the equation of state (3.4) we will consider the equation of state of an ideal gas

$$
p=c^{2} \rho
$$

where $c$ is the isothermal speed of sound.

With the particular value $\sigma=0$ in (3.1) we obtain the so-called explicit completely conservative scheme [2]. Equation (3.2) of this scheme is not explicit, its right side contains the values of the mesh function of velocity from the top time layer $v_{i}^{j+1}$. However, this scheme can be solved explicitly without difficulty. For this we have to consider Eqs. (3.1)-(3.4) in the sequence in which they are written. Then from Eq. (3.1) for the known values of the mesh pressure function $p_{i}{ }^{j}$ we determine the velocity on the $(j+1)$-th layer $v_{i}^{j+1}$; then from Eq. (3.2) we determine $x_{i}^{j+1}$, from Eq. (3.3) the function $\rho_{i}^{j+1}$, and from Eq. (3.4) the pressure $p_{i}^{j+1}$.

The scheme described, attractive from the algorithmic point of view, is stable in the acoustic approximation only when the condition [2]

$$
\tau \leqslant K h^{2}
$$

is satisfied, where $K$ is some constant. Then the computational errors may increase with time, but not more rapidly than $e^{K t / 3}$.

The stability condition (3.5) imposes on the mesh step $\tau$ a constraint which is rigid and unnatural for hyperbolic equations. It does not permit the use for calculations of a mesh with a coarse time step, and therefore it does not make it possible to realize the advantages possessed by completely conservative schemes over other schemes, since these advantages emerge on coarse meshes.

\section{The simplest iterative process}

For the parameter values $\sigma \geqslant 0.5$ the scheme (3.1)-(3.4) is unconditionally stable $[2,7]$. However, in this case it has not so far been possible to solve the resulting system of difference equations explicitly. This system of algebraic equations is non-linear, therefore we have to use various iterative methods to solve it.

Here the value of any mesh function $y$ on the top layer is defined as follows: 


$$
y_{i}^{j+1}=\lim _{k \rightarrow \infty} y_{i}^{[k]}
$$

where $k$ is the number of the iteration. It is natural that actual calculations are limited to a finite number of iterations $k_{0}$, using as a practical criterion of the convergence of the iterations some condition of the form

$$
\left|y_{i}^{[k+1]}-y_{i}^{[k]}\right| \leqslant \varepsilon_{1}\left|y_{i}^{[k]}\right|+\varepsilon_{2}, \quad i=0,1, \ldots, N
$$

Here $\epsilon_{1}$ is the relative accuracy, $\epsilon_{2}$ is some small number which ensures correct "operation" of the criterion (4.2) in the particular case $y_{i}^{[k]}=0$.

Naturally, an iterative process does not always converge. The conditions for its convergence generate certain constraints on the time step of the mesh. We consider the nature of these constraints.

We turn to the simplest iterative process for the scheme (3.1)-(3.5) ("explicit iteration"), putting $\sigma=1$ to be specific. The values of the mesh functions at two adjacent iterations the $k$-th and $(k+1)$-th are here connected as follows:

$$
\begin{gathered}
\frac{v_{i}^{[k+1]}-v_{i}^{j}}{\tau}=-\frac{p_{i}^{[k]}-p_{i-1}^{[k]}}{h}, \\
\frac{x_{i}^{[k+1]}-x_{i}^{j}}{\tau}=0.5\left(v_{i}^{[k+1]}+v_{i}^{j}\right) \\
\frac{1}{\rho_{i}^{[k+1]}}=\frac{x_{i+1}^{[k+1]}-x_{i}^{[k+1]}}{h}, \\
p_{i}^{[k+1]}=c^{2} \rho_{i}^{[k+1]} .
\end{gathered}
$$

In exactly the same way as in the case of the explicit conservative scheme considered in section 3, the system of equations (4.3)-(4.6) is solved explicitly at each iteration: from (4.3) we determine $v_{i}^{[k+1]}$, from (4.4) we determine $x_{i}^{[k+1]}$ and so on.

As the "zeroth" iteration $y_{i}[0]$ is usually taken as the value from the preceding time layer $y_{i}^{j}$.

It is shown by analysis [2] that to a linear approximation the process described converges only if the inequalities

$$
\tau \leqslant \tau_{K} / 2^{1 / 2}
$$

are satisfied, where $\tau_{K}=h / c \rho$ is the quantity occurring in Courant's well-known stability condition $\tau \leqslant \tau_{k}$, constructed for the "cross" scheme [8]. 
Therefore, when the simplest iterative process is used the condition that the iterations converge generates a fairly strong constraint on the mesh step $\tau$, although the original scheme is absolutely stable.

\section{Newton's iterative method}

To solve the difference equations of the gas dynamic scheme (3.1)-(3.4) we can use Newton's iterative method, which possesses a rapid rate of convergence. Applying the usual procedure to the system of non-linear algebraic equations (3.1)-(3.4) $[2,9]$, we arrive at each iteration $k=1,2, \ldots$ at a system of linear equations

$$
\begin{aligned}
& \delta v+\sigma \tau \delta p_{s}=-f_{1}, \quad \delta x-0.5 \tau \delta v=-f_{2}, \\
& \delta x_{s}-\frac{1}{\rho^{2}} \delta \rho=-f_{3}, \quad \delta p-c^{2} \delta \rho=0 .
\end{aligned}
$$

In (5.1) the quantity $\delta y$ is the difference between the values of the mesh function $y$ in adjacent iterations the $(k+1)$-th and the $k$-th:

$$
\delta y=\delta y^{[k+1]}=y^{[k+1]}-y^{[k]} .
$$

The remaining mesh functions in (5.1), namely $\rho, f_{1}, f_{2}, f_{3}$, are calculated at the $k$-th iteration:

$$
\begin{aligned}
& f_{1 i}^{[k]}=v_{i}^{[k]}-v_{j}^{i}+\sigma \frac{\tau}{h}\left(p_{i}^{[k]}-p_{i-1}^{[k]}\right)+(1-\sigma) \frac{\tau}{h}\left(p_{i}{ }^{j}-p_{i-1}^{j}\right), \\
& f_{2 i}^{[k]}=x_{i}^{[k]}-x_{i}^{j}-0.5 \tau\left(v_{i}^{[k]}+v_{i}^{j}\right), \quad f_{3 i}^{[k]}=\frac{x_{i+1}^{[k]}-x_{i}^{[k]}}{h}-\frac{1}{\rho^{[k]}} .
\end{aligned}
$$

We note that because of the linearity of Eqs. (3.1), (3.2) we have $f_{1 i}^{\left[h_{1}\right.}=f_{2 i}^{\left[h_{1}\right.}=0$ for $k \geqslant 1$.

The system (5.1) is linear in the unknown increments $\delta y$. Eliminating from (5.1) the functions $\delta p, \delta \rho$ and $\delta x$, we can reduce this system at each node of the mesh to a linear difference equation of the second order in $\delta v$ :

$$
\delta v-0.5 \sigma \tau^{2}\left(c^{2} \rho^{2} \delta v_{*}\right)_{\bar{s}}=-f_{1}+\sigma \tau\left[c^{2} \rho^{2}\left(f_{3}-\left(f_{2}\right)_{*}\right)\right]_{\bar{s}}
$$

or, in subscript form,

$$
A_{i} \delta v_{\imath-1}-C_{i} \delta v_{1}+B_{i} \delta v_{i+1}=-F_{i}, \quad i=1,2 \ldots, N-1 .
$$

The coefficients of Eq. (5.4) depend on the number of the iteration and are calculated by the formulas

$$
\begin{aligned}
& A_{i}=A_{i}^{[k]}=\frac{\sigma}{2}\left(\frac{c \tau}{h}\right)^{2}\left(\rho_{i-1}^{[k]}\right)^{2}, \quad B_{i}=B_{i}^{[k]}=A_{i+1}^{[k]} \\
& C_{i}=C_{i}^{[k]}=1+A_{i}^{[k]}+B_{i}^{[k]}, \\
& F_{i}=F_{i}^{[k]}=-f_{12}^{[k]}+\sigma \tau c^{2}\left\{\left(\rho_{i}^{[k]}\right)^{2}\left[f_{3 i}^{[k]}-\left(f_{2 i}^{[k]}\right)_{s}\right]\right\}_{\overline{-}}
\end{aligned}
$$


The system of three-point equations (5.4) can be solved by pivotal condensation [3] at each iteration.

The stability conditions of the pivotal condensation $A_{i}>0, B_{i}>0, C_{i}>A_{i}+B_{i}$ are here satisfied. The iterative process is continued until some convergence condition, for example of the form (4.2), is satisfied.

\section{Convergence conditions of Newton's iterative method}

We substitute in the system of linearized equations (5.1) in place of the right sides $f_{p}, p=1,2$, 3 , their expressions from (5.3) and then subtract from each equation the corresponding value of the original system (3.1)-(3.4).We obtain

$$
\begin{aligned}
& \Delta v+\sigma \tau \Delta p_{\bar{s}}=0, \quad \Delta x-0.5 \tau \Delta v=0, \\
& \Delta x_{s}+\frac{\rho^{[k+1]}-\rho^{[k]}}{\left(\rho^{[k]}\right)^{2}}-\frac{1}{\rho^{\lfloor k]}}+\frac{1}{\hat{\rho}}=0, \quad \Delta p-c^{2} \Delta \rho=0 .
\end{aligned}
$$

Here $\Delta y=\Delta y^{[k+1]}=y^{[k+1]}-\hat{y}$ is the difference between the value of the mesh function $y$ at the $(k+1)$-th iteration and the solution $\hat{y}=y^{j+1}$. We note that this notation differs from the notation $\delta y$ encountered above (see (5.2)).

The system (6.1) unlike (5.1), is unsuitable for practical calculations, but is convenient for theoretical discussions. After climinating from (6.1) the increments of all the functions except $\Delta \rho$, we can transform this system to the second-order equation:

where

$$
\begin{aligned}
& \left(1+2\left(a_{i}^{[k]}\right)^{2}\right) z_{i}^{[k+1]}-\left(a_{i}^{[k]}\right)^{2}\left(z_{i+1}^{[k+1]}+z_{i-1}^{[k+1]}\right)=-\frac{\left(z_{i}^{[k]}\right)^{2}}{\rho_{i}^{j+1}} \\
& i=1,2, \ldots, N-1
\end{aligned}
$$

$$
z_{i}^{[k+1]}=\Delta \rho_{i}^{[k+1]}, \quad\left(a_{i}^{[k]}\right)^{2}=0.5 \sigma\left(\frac{c \tau}{h}\right)^{2}\left(\rho_{i}^{[k]}\right)^{2} .
$$

If to be specific we confine ourselves to problems where on the boundaries modes of variation of pressure with time are defined, then as boundary conditions we have to supplement Eq. (6.2) by the relations

$$
z_{0}^{[k+1]}=0, \quad z_{N}^{[k+1]}=0, \quad k=0,1, \ldots
$$

For an inhomogeneous equation (6.2) with homogeneous boundary conditions (6.3) the maximum principle holds $[2,3]$, which implies the corollaries

Here

$$
\begin{gathered}
z_{i}^{[k+1]}=\rho_{i}^{[k+1]}-\rho_{i}^{j+1} \leqslant 0 \\
\left\|z_{i}^{[k+1]}\right\|_{c} \leqslant\left\|\frac{\left(z_{i}^{[k]}\right)^{2}}{\rho^{j+1}}\right\|_{c} \leqslant\left\|\frac{1}{\rho^{j+1}}\right\|_{c}\left\|z^{[k]}\right\|_{c}^{2} .
\end{gathered}
$$

$$
\left\|z^{[k]}\right\|_{c}=\max _{i}\left|z_{i}^{[k]}\right|
$$


The inequality (6.4) means that in the iterative process the approach to the solution $\rho_{i}^{j+1}$ goes from below. After many applications condition (6.5) reduces to the form [2]

$$
\left\|z^{[k+1]}\right\|_{c} \leqslant q^{2 k+1}-1\left\|z^{[0]}\right\|_{c}
$$

where

$$
q=\left\|1 / \rho^{i+1}\right\|_{c}\left\|z^{[0]}\right\|_{c}=\left\|1 / \rho^{j+1}\right\|_{c}\left\|\rho^{j+1}-\rho^{[0]}\right\|_{c}>0
$$

It is obvious that the iterative process converges if $q<1$.

If as the zeroth iteration we take the values of the mesh functions from the preceding $j$-th time layer, as is usually done in practice, then the condition of convergence of the iterations can be written as follows:

$$
q=\tau\left\|\eta^{j+1}\right\|_{c}\left\|\rho_{t}\right\|_{c}<1
$$

$\eta=1 / \rho$. is the specific volume.

Condition (6.7), being sufficient, is obtained for the non-linear case (in the linear approximation the Newton process converges after one iteration) and has an extremely general form, depending on the nature of the solution. However, it is obvious that for any solution condition (6.7) can be satisfied by decreasing the mesh step $\tau$.

For solutions strongly varying with time the constraints on $\tau$ will be strict.

\section{Estimation of the rate of convergence of the iterations for the shock wave problem}

We will explain the constraints to which the general condition of convergence of the iterations (6.7) leads in the problem of a shock wave, "blurred" by viscosity. This example is important for applications, since in actual calculations, in order to ensure the possibility of straight through calculation of shock waves without the explicit isolation of their fronts on the mesh artificial viscosity is introduced into the scheme. This viscosity $\omega$ occurs as an addition to the gas-dynamic pressure $p$. The linear viscosity has the form

$$
\omega=-v \rho \partial v / \partial s
$$

$\nu$ is the viscosity coefficient.

The solution of the problem of the structure of the front of an isothermal shock wave in a viscous medium is known (see, for example, $[1,2]$ ). It is usually described by the ordinary differential equation

$$
\frac{d \eta}{d \zeta}=\frac{D}{v}\left(\eta_{0}-\eta\right)\left(\eta-\eta_{1}\right)
$$


Its solution is

$$
\eta(\zeta)=\left\{\eta_{1}+K \eta_{0} \exp \left[\frac{D\left(\eta_{0}-\eta_{1}\right)}{v}\right] \zeta\right\}\left\{1+K \exp \left[\frac{D\left(\eta_{0}-\eta_{1}\right)}{v}\right] \zeta\right\}^{-1}
$$

where $\zeta=s-D t$ is a self-similar variable, $D$ is the mass velocity of the shock wave front, $\eta_{0}, \eta_{1}$ are the values of the specific volume ahead of the wave and after it respectively, and $K$ is an arbitrary constant. The value of $\eta_{0}$ is specified, the value of $\eta_{1}$ is defined in the isothermal case by the formula $\eta_{1}=c^{2} \rho_{0} / D^{2}=\alpha^{2} \eta_{0}$, where $\alpha=m / D<1 \quad$ is the ratio of the mass speed of sound $m=c \rho_{0}$, to the mass speed of the shock wave front. Figure 1 shows the solution of the problem of the structure of the shock wave in Lagrangian mass coordinates at a fixed instant. This solution enables us to estimate the values of the norms of the functions occurring in the convergency condition (6.7):

$$
\left\|\eta^{\prime+1}\right\|_{c}=\eta_{0}, \quad\left\|\rho_{t}\right\|=\frac{D^{2}}{4 v} \frac{\left(\eta_{0}-\eta_{1}\right)^{2}}{\eta_{0} \eta_{1}}
$$

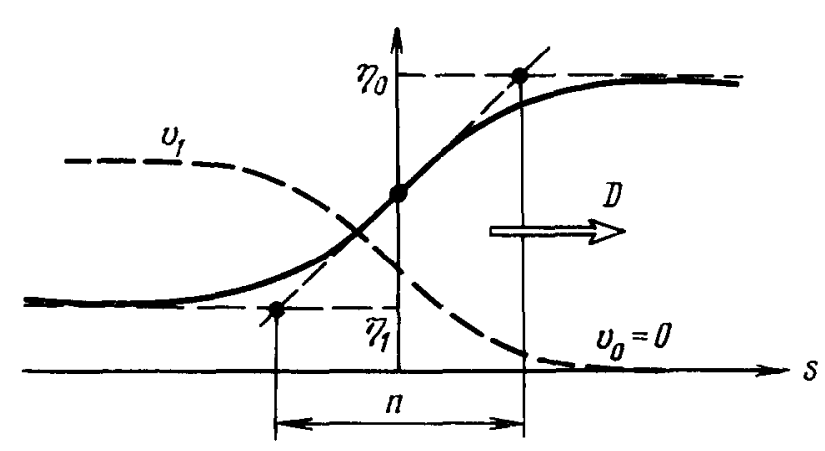

FIG. 1.

After substituting the relations (7.3) in the condition of convergence of the iterations (6.7) the latter is reduced to the form

$$
\xi=\frac{\tau}{\tau_{K 1}}<n \Phi(\alpha)=n \frac{\alpha}{1-\alpha^{2}}
$$

where $\tau_{K_{1}}=h /\left(c \rho_{1}\right)$ is the quantity calculated by Courant's stability condition for the parameters behind the shock wave front, $\alpha=m / D, 0<\alpha \leqslant 1$, is the characteristic intensity of the shock wave, and $n$ is the number of mass intervals of the mesh on which the shock wave front is blurred.

Therefore, the size of the maximal step $\tau$ depends on the strength of the wave (on the value of the parameter $\alpha$ ). Strong shock waves with $\alpha \rightarrow 0$ require a small step; for the calculation of weak waves, close to acoustic $(\alpha \rightarrow 1)$, there are practically no constraints.

It is seen that the convergence conditions of the Newton method are noticeably less strict than in the method of simple iterations considered in section 4 . 


\section{Results of numerical calculations}

The estimates obtained above of the step $\tau$ permissible for convergence of the iterations are confirmed by numerical calculations. As a test problem we considered the classical problem of a piston moved in a gas with constant velocity, as a result of which a shock wave arises.

In the calculations we consider a finite mass of gas $0 \leqslant s \leqslant M$, bounded on the left at $s=0$ by the piston, whose velocity is given:

$$
v(0, t)=U
$$

and on the right $(s=M)$ by a fixed wall:

$$
v(M, t)=0
$$

The quantity $M$ is chosen to be so large that the shock wave mode is successfully formed. The calculation is continued until the shock wave front reaches the right boundary. The initial state of the gas was of the form

$$
\rho(s, 0)=\rho_{0}=1, \quad p(s, 0)=p_{0}=0.25, \quad v(s, 0)=v_{0}=0, \quad 0<s<M .
$$

All the reasoning applies to the isothermal case, the speed of sound $\left(\right.$ see $\left.\left(3.4^{\prime}\right)\right) c=0.5$.

A comparative analysis of various schemes was carried out on the example of a version of the problem, where the velocity of the piston (8.1) was $U=0.75$. Then, as follows from the Hugoniot relations, the speed of the shock wave front $D=1$, and the gas parameters behind the front have the following values:

$$
\rho_{1}=4, \quad \eta_{1}=0.25, \quad p_{1}=1, \quad v_{1}=0.75 .
$$

In calculations in the region $0 \leqslant s \leqslant M, M=3$ to 7 , a uniform mesh with step $h=0.1$ was introduced. The time step $\tau$ of the mesh was varied.

To ensure the possibility of a straight through calculation of the shock wave in the scheme the linear viscosity (7.1) with coefficient $\nu$ was introduced, so that the wave front was blurred on $n$ mesh intervals:

$$
n=\frac{4 v}{D h\left(\eta_{0}-\eta_{1}\right)}
$$

For $v=0.05$ (this is the value of the viscosity coefficient used in the calculations) and $D=1$ we have $n=2.6$.

Figure 2 shows the results of a calculation (the velocity profiles at two consecutive instants) of the piston problem, obtained by the explicit scheme (3.1)-(3.4), $\sigma=0$, for various values of the $\tau$ step. For $\tau=0.03, \xi=\tau / \tau_{K 1}=0.6$ the solution goes well (Fig. $2, a$ ), for $\tau=0.04, \xi=0.8$ the profile of $v$ behind the wave front becomes "spiky" (Fig. 2,b). For larger values $\tau \sim \tau_{K_{1}}=0.05$, $\xi \sim 1$ the developing "bump" completely distorts the solution (Fig. 2,c). Therefore, the use of the explicit difference scheme in this problem is efficient only for a value of the mesh step $\tau \leqslant \tau_{k 1}, \xi \leqslant 1$. 


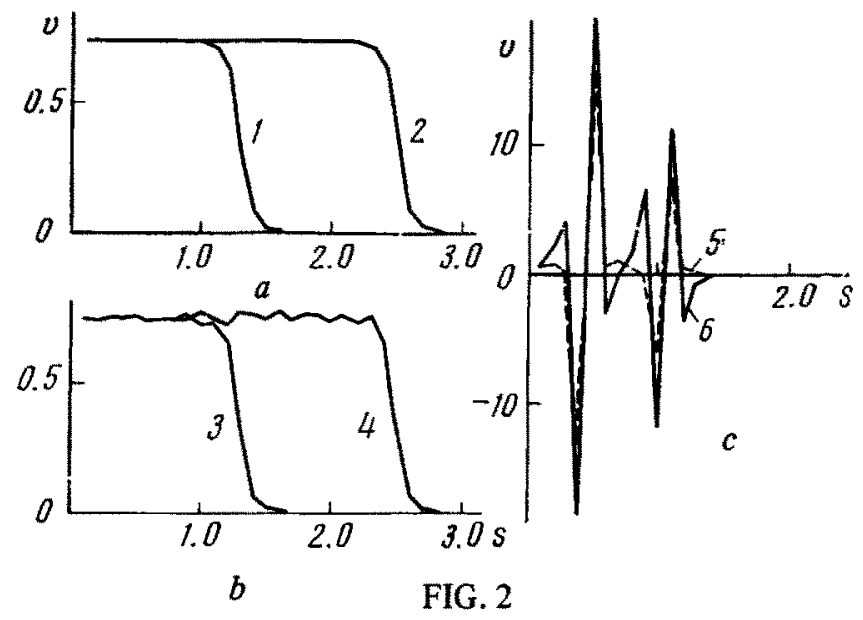

1 is for $t=1.2, j=40 ; 2$ is for $t=2.4, j=8 ; 3$ is for $t=1.2, j=30 ; 4$ is for $t=2.4, j=60$; 5 is for $t=0.5, j=10 ; 6$ is for $t=1, j=20$.

Figure 3 gives the results of the calculation of the same problem by the purely implicit scheme of (3.1)-(3.4), $\sigma=1$, using an explicit iterative process (see section 4). For $\tau=0.015, \xi=0.3$ (see Fig. 3,a) the solution goes satisfactorily, for $\tau=0.0175, \xi=0.35$ (see. 3,b) the velocity profile acquires a pronounced "spiky" nature. We note that already for some values of $\tau$ the iterations converge badly. In the calculations the number of iterations per step was restricted to the value $k_{0}=30$ for $\tau=0.015$ and $k_{0}=100$ for $\tau=0.0175$. Nevertheless this number of iterations was insufficient to satisfy the convergence condition (4.2) with the relative accuracy $\varepsilon_{1}=10^{-6}$.

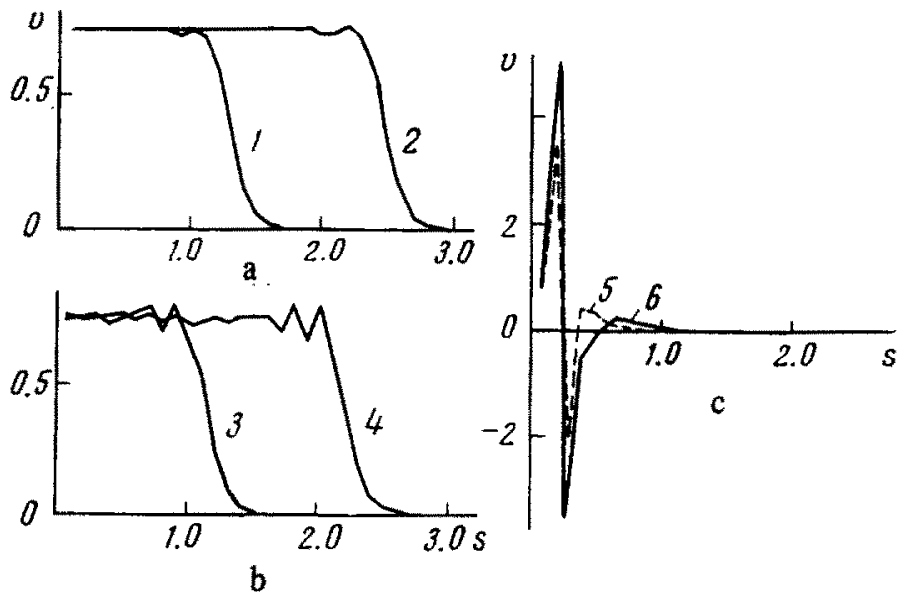

FIG. 3

1 is for $t=1.2, j=80 ; 2$ is for $t=2.4, j=160 ; 3$ is for $t=0.5, j=60 ; 4$ is for $t=2.1, j=120$; 5 is for $t=0.4, j=20 ; 6$ is for $t=0.8, j=40$. 
When the step size $\tau$ is increased further, for example for $\tau=0.02, \xi=0.4$ (see Fig. 3,c), it was not possible to reproduce the solution by means of this algorithm.

Therefore, we obtained experimentally the constraint on the mesh step

$$
\tau \leqslant 0.3 \tau_{K 1}, \quad \xi \leqslant 0.3 \text {, }
$$

on the satisfaction of which the algorithm satisfactorily gives the solution of the piston problem. We recall that the condition of convergence of the iterations obtained above theoretically for the acoustic case, has the form

$$
\tau \leqslant \tau_{K_{1}} / 2^{1 / 2}, \quad \xi \leqslant 0.7 \text {. }
$$

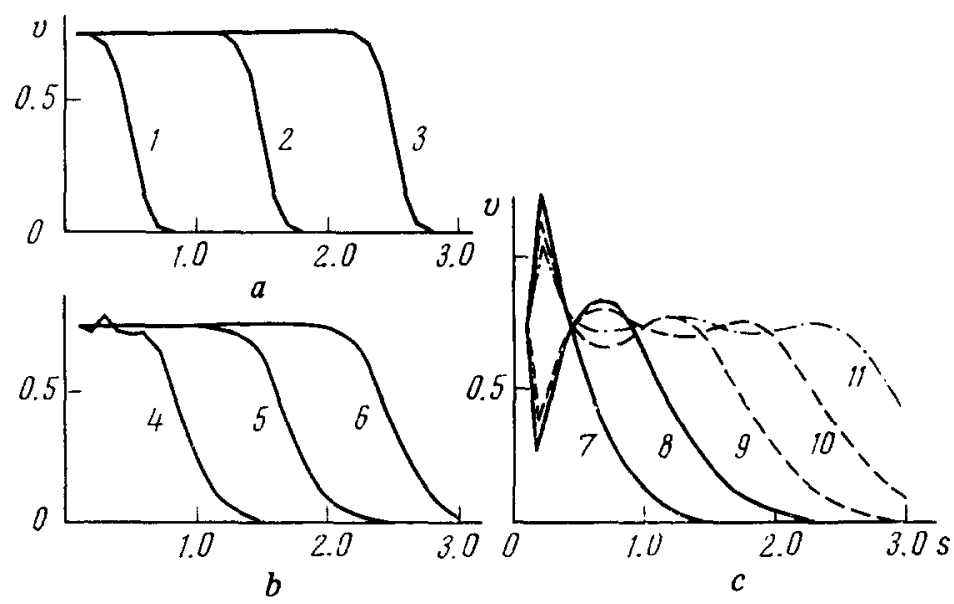

FIG. 4

1 is for $t=0.4, j=40 ; 2$ is for $t=1.4, j=140 ; 3$ is for $t=2.4, j=240 ; 4$ is for $t=0.8, j=4$; 5 is for $t=1.6, j=8 ; 6$ is for $t=2.4, j=12 ; 7$ is for $t=0.6, j=1 ; 8$ is for $t=1.2, j=2 ; 9$ is for $t=1.8$, $j=3 ; 10$ is for $t=2.4, j=4 ; 11$ is for $t=3, j=5$.

Figure 4 gives the results of calculations performed by the purely implicit scheme of (3.1)-(3.4), $\sigma=1$, using Newton's iterative method for the following values of the step $\tau: \tau=0.01, \xi=0.12$ (see Fig. 4,a); $\tau=0.2, \xi=4$ (see Fig. 4,b); $\tau=0.6, \xi=12$ (see Fig. 4,c).

We note that the step $\tau=0.6$ is coarse, in this calculation the wave front passes in one time step through six mass intervals of the mesh. Nevertheless the solution is reproduced satisfactorily and the number of iterations per step is small $(k=2$ for $\tau=0.01, k=3$ for $\tau=0.02, k=4$ for $\tau=0.6)$.

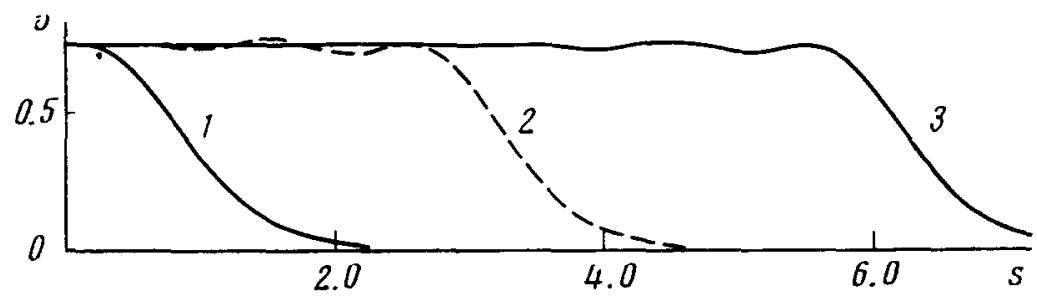

FIG. 5

1 is for $t=0, j=0 ; 2$ is for $t=2.4, j=4 ; 3$ is for $t=5.4, j=9$. 
A drawback of the last series of calculations is the oscillations which the functions undergo near the piston. The oscillations are observed only for a fairly large value of the mesh time step for $\tau \geqslant 0.3, \xi>6$ (see Fig. 4,c). They occur in time from step to step with decreasing amplitude. These oscillations are a consequence of the "first shock" which the piston produces at the initial instant, moving a considerable distance in one step for such large values of $\tau$. This is verified by the results of the calculations shown in Fig. 5. Here the values of all the parameters are the same as for the calculations in Fig. 4,c, except for the initial data: for Fig. 4,c they are given by Eqs. (8.3), for Fig. 5 they are the smooth distributions corresponding to the "blurred" shock wave, advanced to $t=0$ from the piston after 10 mass intervals. As is obvious from Fig. 5 , the further motion of the shock wave for $t>0$ is not accompanied by oscillations of the functions near the piston. It is obvious that we would have obtained the same result if the piston had acquired the velocity $U$ gradually, and not acquired it instantaneously at $t=0$, as occurred in the given calculations.

Another type of oscillation which is observed in calculations with coarse values of $\tau$, is the wavelike distribution of parameters behind the shock front (see Fig. 4, 5). The amplitude of this wave increases with $\tau$, at th same time the wave dies away with distance from the front to the piston. The distance between the maximum and the minimum in the profile formed behind the shock front (half the wavelength) equals the number of mass intervals of the mesh which the front traverses in one time step (for Figs. 4,c and 5 this number equals six intervals).

The reason for the occurrence of these oscillations is the discrete nature of the medium (difference mesh) through which the shock wave moves.

\section{Approximation viscosity}

A characteristic detail of the series of calculations shown in Fig. 4 is the features of the blurring of the shock wave front. For small values of $\tau$ the effective width of the wave front in the calculations is the same as the value (3.4) obtained for the exact solution. However, as $\tau$ increases this width grows, although the value of the viscosity in (7.1) remains unchanged (see Fig. 6, which shows profiles of the specific volume and viscosity in the steady shock wave obtained in calculations for various values of $\tau$ ).
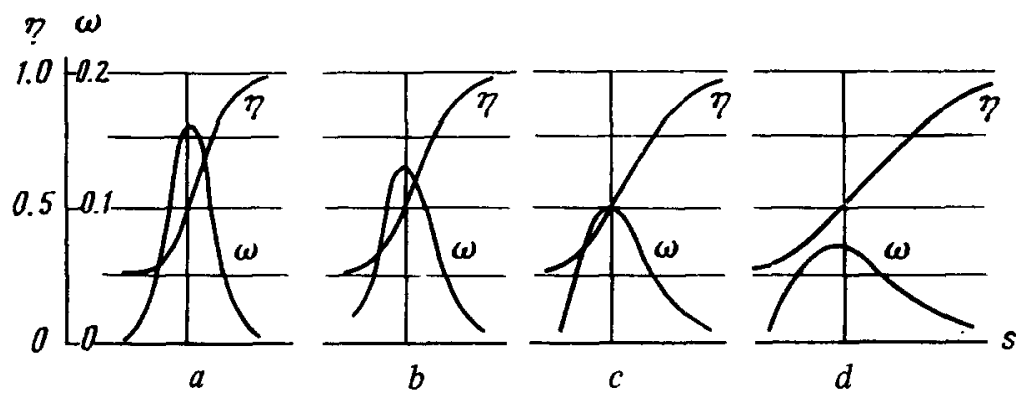

FIG. 6

$a$ is for $\tau=0.1, b$ is for $\tau=0.2, c$ is for $\tau=0.35, d$ is for $\tau=0.7$.

To explain the cause of this phenomenon we represent the difference equation of motion of the scheme 


$$
v_{t}=-g \frac{(\sigma)}{3}, \quad g=p+\omega
$$

in the form

$$
v_{t}=-\left(g^{(0.5)}+(\sigma-0.5) \tau g_{t}\right)_{\bar{s}} .
$$

Here we have used the difference formula [2]

$$
y^{(\alpha)}=y^{(\beta)}+(\alpha-\beta) \tau y_{t}
$$

It is obvious that for any value of $\sigma$ the scheme of (9.2) has the second-order approximation $O\left(\tau^{2}+h^{2}\right)$ with respect to the point $\left(s_{i+1 / 2}, t_{j+y_{3}}\right)$.

We may consider formally that the difference equation (9.2) approximates with second order the differential equation

$$
\frac{\partial v}{\partial t}=-\frac{\partial}{\partial s}\left(g+(\sigma-0.5) \tau \frac{\partial g}{\partial t}\right) \text {. }
$$

This equation corresponds to a continuous medium, where, besides the usual viscosity $\omega$ there also acts some additional viscosity

$$
\bar{\omega}=(\sigma-0.5) \tau \partial g / \partial t .
$$

The coefficient of this viscosity depends on the parameters of the discrete medium $\sigma$ and $\tau$, where as $\tau \rightarrow 0$ the additional viscosity $\bar{\omega}$. becomes vanishing small.

The quantity $\bar{\omega}$ may also be regarded as a term of order $O(\tau)$ of the error of approximation $\psi=O\left(\tau+h^{2}\right)$ of the ordinary differential equation of motion

$$
\partial v / \partial t=-\partial g / \partial s
$$

by the difference equation (9.1).

Thus, $\bar{\omega}$, causing additional dissipative properties of the medium, is part of the so-called approximation viscosity $[10]$, that is, the characteristic viscosity of the scheme.

For a scheme with $\sigma=1$, which is also considered above, we have $\bar{\omega}=-0.5 \tau \partial g / \partial t$.

In the problem of the structure of the front of an isothermal shock wave, where the viscosity $\bar{\omega}$ generates additional blurring of the wave, the viscous terms $\omega$ and $\bar{\omega}$ can be represented by means of the exact solution of (7.2) in the form

$$
\begin{aligned}
& \omega=\frac{\nu}{\eta} D \frac{d \eta}{d \zeta}, \\
& \bar{\omega}=\frac{\tau}{2} D^{3} \frac{d \eta}{d \zeta} .
\end{aligned}
$$

Therefore, for the problem considered the function $\quad \Omega=\omega+\bar{\omega} \quad$ may be regarded as some total viscosity

$$
\Omega=\frac{\nu+0.5 \tau D^{2} \eta}{\eta} D \frac{d \eta}{d \zeta}
$$


which is linear and possesses the effective coefficient

$$
\bar{v}=v+0.5 \tau D^{2} \eta \text {. }
$$

This coefficient depends on $\eta$, but for estimation we can use also some mean value

$$
\bar{v}_{2 \Phi}=v+0.5 \tau D^{2} \bar{\eta} \text {, }
$$

where $\bar{\eta}$ is some effective value of the specific volume $\eta_{1} \leqslant \bar{\eta} \leqslant \eta_{0}$. It follows from (8.4) that the width of blurring of the wave front by the total viscosity $\Omega$, will be defined by the formula

$$
\bar{n}(\tau)=\frac{4 \bar{v}_{s \phi}}{D h\left(\eta_{0}-\eta_{1}\right)}=\frac{4\left(v+0.5 \tau D^{2} \bar{\eta}\right)}{D h\left(\eta_{0}-\eta_{1}\right)}
$$

As is obvious from (9.3), as $\tau \rightarrow 0$ the quantity $\bar{n}$ is identical with the classical value (8.4), which follows from the exact solution of the differential problem of the structure of the shock wave front. As $\tau$ increases the width of blurring increases linearly.

Calculations confirm this result. Figure 7 shows the variation of $\bar{n}(\tau)$ obtained experimentally as a result of processing a series of calculations, some of which are represented by circles. Here the values of the parameters are as follows:

$$
D=1, \quad v=0.05, \quad h=0.1, \quad \eta_{0}=1, \quad \eta_{1}=0.25 \text {. }
$$

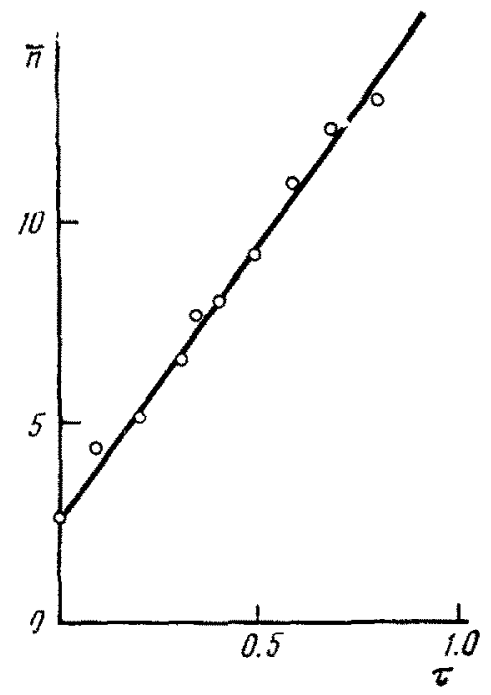

FIG. 7

The experimental points in Fig. 7 lie well on the straight line (4.3) with $\bar{\eta} \approx 0.5$. This is natural, since calculations show that the action of viscosity is a maximum in a region with $\eta \approx 0.5$ (Fig. 6).

Therefore, because of the presence of approximation viscosity, the sufficient condition for the convergence of the iterations (7.4) obtained above must contain, instead of the fixed width of blurring of the shock wave front, the quantity $\bar{n}(\tau)$ increasing with $\tau$. 
This, and also the fact that the condition of convergence of the iterations (7.4) is of a sufficient nature, permits the use in practice of meshes with steps $\tau$ noticeably exceeding the maximum value prescribed by the inequality (7.4).

It must be mentioned that all the reasoning in section 9 is of an approximate nature, since it is based on Eq. (7.4), obtained for scheme not taking viscosity into account, while viscosity is present in the calculations.

In conclusion the authors thank N. N. Tyurina for performing the numerical calculations.

Translated by J. Berry

\section{REFERENCES}

1. ROZHDESTVENSKII, B. L. and YANENKO, N. N. Systems of quasilinear equations (Sistemy kvazilineinykh uravnenii), "Nauka", Moscow, 1968.

2. SAMARSKII, A. A. and POPOV, Yu. P. Difference schemes of gas dynamics (Raznostnye skhemy gazovoi dinamiki), "Nauka", Moscow, 1975.

3. SAMARSKII, A. A. Introduction to the theory of difference schemes (Vvedenie $\mathrm{v}$ teoriyu raznostnykh skhem), "Nauka", Moscow, 1971.

4. POPOV, Yu. P. and SAMARSKII, A. A. Completely conservative schemes. Zh. vychisl. Mat. mat. Fiz., 9, $4,953-958,1969$.

5. RICHTMYER, R. D. Difference methods of solving boundary value problems (Raznostnye metody resheniya kraevykh zadach), Izd-vo in. lit., Moscow, 1960.

6. SAMARSKII, A. A., VOLOSEVICH, P. P., VOLCHINSKAYA, M. I. and KUDRYUMOV, S. P. A finitedifference method for the solution of one-dimensional non-stationary problems in magneto-hydrodynamics. Zh. vȳchisl. Mat. mat. Fiz., 8, 5, 1025-1038, 1968.

7. SAMARSKII, A. A. and GULIN, A. V. Stability of difference schemes (Ustoichivost raznostnykh skhem), "Nauka", Moscow, 1973.

8. COURANT, R., FRIEDRICHS, K. and LEVY, H. The difference equations of mathematical physics. Usp. mat. Nauk, 8, 125-160, 1940.

9. BEREZIN, I. S. and ZHIDKOV, N. P. Computing methods (Metody vychislenii), Vol. 2, Fizmatgiz, -Moscow, 1962.

10. YANENKO, N. N. and SHOKIN, Yu. I. On the approximation viscosity of difference schemes. Dokl. Akad. Nauk SSSR, 182, 4, 776-778, 1968. 